\title{
Tratamento da Artrite Reumatóide
}

\author{
Rheumatoid Arthritis Treatment
}

\author{
Maria Raquel da Costa Pinto ${ }^{(1)}$, Rita de Cássia Corrêa Miguel ${ }^{(2)}$, Gustavo Gomes Rezende $^{(2)}$
}

\begin{abstract}
A artrite reumatóide $(A R)$ éa doença que identifica a especialidade. $H$ istoricamente, é entendida como uma condição clínica potencialmente grave, não só pela morbidade associada à dor, à limitação funcional eà repercussão na qualidade de vida dos pacientes, mas também por mortalidade maior em relação à população geral. No entanto, em sua missão de assistir aos pacientes com AR, o médico reumatologista tem hoje o privilégio de viver um momento de grande desenvolvimento. $N$ ovas alternativas terapêuticas se confirmam mais específicas e eficazes, trazendo ao nosso vocabulário palavras como remissão e cura. M as também é importante mencionar que o melhor conhecimento deve ser acompanhado de maior compromisso e a nossa atuação deve ser cada vez mais pautada pela ética e responsabilidade nas decisões so bre o tratamento dos nossos pacientes. Esta seção traz uma seleção comentada de artigos originais discutindo os aspectos mais atuais do tratamento daAR.
\end{abstract}

Kapral T, Stamm T, M achold KP, M ontag K, Smolen J S, Aletaha D: M ethotrexate in rheumatoid arthritis is frequently effective, even if re-employed after a previous failure ( $M$ etotrexato na artrite reumatóide é freqüentemente eficaz, mesmo se reempregado após fracasso prévio). Arthritis Res Ther 8: R 46, 2006. M edical University of Vienna, Viena, Áustria.

O s autores analisaram a eficácia do reemprego de metotrexato (M TX) nos pacientes com história de falha prévia a esta droga no tratamento de AR. D entre os 1490 pacientes de uma coorte histórica, 163 submeteram-se ao reemprego do MTX, definido como novo curso após interrupção, com o uso de pelo menos uma outra droga modificadora da doença no intervalo, excluindo-se terapias combinadas. As doses de M TX prescritas no reemprego foram maiores que no uso original (medianas de $15 \mathrm{e}$
10 mg respectivamente; $p<0,001)$. D os pacientes inicialmente considerados refratários, $45 \%$ obtiveram sucesso com a reintrodução. 0 fator preditivo de boa resposta mais importante foi o uso anterior de doses mais baixas. A freqüência de efeitos adversos foi similar. 0 trabalho concluiu que, especialmente quando doses baixas de M TX foram empregadas num curso prévio interrompido por ineficácia, a reintrodução de M TX pode ser uma opção com boas chances de sucesso.

Grigor C, Capell H, Stirling A et al: Effect of a treatment strategy of tight control for rheumatoid arthritis (the TICORA study): a single-blind randomised controlled trial (E feito de uma estratégia de tratamento de controle rígido para artrite reumatóide - estudo TI CO RA: um ensaio randomizado, controlado, simples-cego). Lancet 364: 263-9, 2004. Gartnavel General H ospital, Glasgow, Reino U nido.

Este estudo testou duas modalidades de manejo de pacientes com AR: um grupo de tratamento intensivo com visitas mensais e um grupo controle. Para os pacientes do grupo de tratamento intensivo, a cada visita em que houvesse persistência de atividade moderada ( $D A S>2,4$ ) era feito o escalonamento de drogas modificadoras da doença (D M ARD s) até que se obtivesse redução da ativi- dade ou aparecimento de toxicidade. 0 protocolo iniciou sulfassalazina (SSZ) em doses semanalmente progressivas com acréscimo subseqüente de hidroxicloroquina mais M TX, em doses com incrementos semanais, e folato. A pós isto, associava-se prednisolona $7,5 \mathrm{mg} /$ dia e trocava-se a SSZ por ciclosporina, caso fosse mantido DAS $>2,4$. Por fim, poderia haver troca do esquema combinado para

1. Serviço de Reumatologia do Hospital das Clínicas da Universidade Federal de Minas Gerais (UFMG) e do Hospital Socor de Belo Horizonte (MG).

2. Residentes do Serviço de Reumatologia do Hospital das Clínicas da UFMG. 
leflunomida ou aurotiomalato de sódio. Além disso, os pacientes do grupo intensivo receberam corticosteróides (CE) intra-articular e/ ou intramuscular. No grupo controle, as visitas foram trimestrais e não houve avaliação formal de atividade que guiasse as decisões terapêuticas. A queda média no escore de atividade foi maior no grupo intensivo que no controle (-3,5 versus -1,9; diferença de 1,6 [ CI 95\% $1,1-2,1] p<0,0001)$. A proporção de pacientes com boa resposta segundo os critérios da European L eague Against Rheumatism (EU LAR) também foi maior no grupo intensivo ( $82 \%$ vs $44 \%$, OR 5,8 [ $C I 95 \% 2,4-13,9] \mathrm{p}<0,0001$ ). A contagem de erosõese o escore de dano radiológico de Sharp total foram menores no grupo intensivo $(0,5$ vs $3 p=0,002$ e 4,5 vs 8,5 p=0,02), mas o escore de redução de espaço articular não alcançou diferença estatística. O s autores concluíram que os principais fatores que levaram aos resultados mais favoráveis com a estratégia de tratamento intensivo foram: maior número de pacientes usando terapia combinada, doses maiores de M TX, CE intra-articular e menor taxa de abandono (garantida pela melhor educação do paciente e maior relação de confiança com visitas mais freqüentes). Os resultados demonstraram ainda que os custos em saúde não aumentaram, pois o maior número de consultas e exames foi compensado pela menor quantidade de internações.

Korpela M , L aasonen L, H annonen $\mathrm{P}$, et al (FIN -RAC o Trial G roup): Retardation of joint damage in patients with early rheumatoid arthritis by initial aggressive treatment with disease-modifying antirheumatic drugs: five-year experience from the FIN-RACo study. (Atraso no dano articular em pacientes com artrite reumatóide inicial através de tratamento inicial agressivo com drogas anti-reumáticas modificadoras da doença: experiência de cinco anos do estudo FIN -RACo). Arthritis Rheum 50: 2072-81, 2004. Tampere U niversity H ospital, Tampere, Finlândia.

O s autores analisaram as taxas de resposta clínica, taxas de remissão e progressão radiológica de pacientes com $A R$ inicial, definida como menos de dois anos de sintomas, em um estudo multicêntrico, prospectivo $(n=160)$. I nicialmente 78 pacientes foram randomisados para receber terapia combinada (M TX, SSZ ehidroxicloroquina mais prednisolona) e 82 para receber monoterapia, (inicialmente com SSZ, havendo possibilidade de troca para M TX ou azatio prina, com ou sem prednisolona). O s pacientes de ambos os grupos nunca haviam usado previamente nenhuma droga modificadora da doença. A pós dois anos, permitiu-se tratar os dois grupos sem restrições, seja com monoterapia ou terapia combinada com ou sem corticosteróide sistêmico. A remissão clínica foi maior no grupo combinado em relação à monoterapia ao final de dois anos ( $40 \%$ versus $18 \% ; p<0,009$ ), mas aos cinco anos, a diferença não alcançou significância estatísti- ca ( $28 \%$ vs 22\%; $p=0,41)$. As médias de DAS28 seguiram esta mesma tendência. 0 número de erosões e o índice radiológico de $L$ arsen foram menores no grupo de terapia combinada aos dois anos e mantiveram diferença estatística até os cinco anos (4 vs $12[p=0,005]$ e 11 vs $24[P=0,001]$, respectivamente). Os autores concluíram que revogar a terapia combinada após dois anos não foi prudente, visto que para os pacientes do grupo combinado, parte da alta taxa de remissão se perdeu após a suspensão das restrições no tratamento ( $40 \%$ aos dois anos e $28 \%$ aos cinco anos). Além disso, a introdução tardia de combinações não aumentou a taxa de remissão em pacientes inicialmente tratados com monoterapia (de $18 \%$ para $22 \%$ ) e nem diminuiu a progressão radiológica em relação àqueles tratados desde o início com terapia combinada. 0 s resultados apresentados reforçam o conceito de janela terapêutica da AR.

Goekoop-Ruiterman YPM, Vries-B ouwstra JK, Allaart CF et al: Clinical and radiographic outcomes of four different treatment strategies in patients with early rheumatoid arthritis (the best study). A randomized, controlled trial (Seguimento clínico e radiográfico de quatro estratégias diferentes de tratamento em pacientes com AR inicial. U m estudo randomizado controlado). Arthritis Rheum 52: 3381-90, 2005. L eiden U niversity Medical Center, L eiden, $\mathrm{H}$ olanda.

N este estudo multicêntrico, os autores comparam resultados clínicos e radiológicos em indivíduos com $A R$ de duração menor ou igual a dois anos, submetidos a diferentes estratégias de tratamento. 0 s pacientes $(n=508)$ foram randomizados em quatro grupos. G rupo 1 ( $n=126)$ : monoterapia seqüencial com drogas anti-reumáticas modificadoras da doença. Grupo 2 ( $n=121$ ): terapia combinada do tipo step up. Grupo 3 ( $n=133$ ): terapia combinada inicial e prednisona em altas doses com redução progressiva. Grupo 4 ( $n=128)$ : terapia combinada inicial com 
infliximabe. Ajustes no tratamento foram feitos a cada três meses, sendo que a meta foi atingir e sustentar um escore de atividade da doença em 44 articulações (D AS44) menor ou igual a 2,4. Após um ano, 53\% (grupo 1), 64\% (grupo 2), 71\% (grupo 3) e 74\% (grupo 4) dos pacientes atingiram esta meta ( $p=0,004$ para grupo 1 versus grupo 3 e $p=0,001$ para grupo 1 vs grupo 4). O s escores médios do HAQ aos três meses foram de 1,0 nos grupos 1 e 2 e de 0,6 nos grupos 3 e 4 ( $p<0,001$ ). A pós um ano, esses escores foram de 0,7 nos grupos 1 e 2 e de 0,5 nos grupos 3 e $4(p=0,009)$. Q uando analisadas as radiografias após um ano de seguimento, foi observada menor progressão de lesão articular nos pacientes dos grupos 3 e 4.0 aumento médio no escore total de Sharp/ Van der $\mathrm{H}$ eijde foi de 2, 2,5, 1 e 0,5 nos grupos 1 a 4, respectivamente. $\mathrm{N}$ ão houve diferença significativa no número de efeitos adversos e interrupção de tratamento entre os grupos. O s autores concluem que, durante o primeiro ano de seguimento, pacientes com diagnóstico de AR precoce que receberam terapia inicial combinada, tanto com prednisona quanto com infliximabe, obtiveram melhora funcional mais rápida e houve menor progressão radiográfica de lesões articulares em comparação aos pacientes que receberam monoterapia ou terapia combinada do tipo step up.

Svensson B, Boonen A, Albertsson K, Van der H eijde D , Keller C, H afström I, for the BARFOT Study G roup: Low-dose prednisolone in addition to the initial disease-modifying antirheumatic drug in patients with early active rheumatoid arthritis reduces joint destruction and increases the remission rate. A two-year randomized trial (Baixas doses de prednisolona em adição ao tratamento inicial com D M ARD na artrite reumatóide recente reduz destruição articular e aumenta taxa de remissão). Artrhitis Rheum 52: 3360-70, 2005. U niversity of L und, L und, Suécia.

Este foi um estudo multicêntrico, randomizado, aberto, com duração de dois anos, desenhado com o objetivo de avaliar a eficácia da prednisolona no dano articular e na atividade da doença, em pacientes com AR com menos de um ano de início de sintomas. Foram incluídos 250 indivíduos com doença ativa (D AS28>3), que não haviam usado previamente corticosteróides ou D M ARD s. O s pacientes foram divididos em dois grupos, sendo que 119 receberam prednisolona na dose de 7,5 $\mathrm{mg} / \mathrm{dia}$ associada a DMARD e 131 receberam apenas DMARD. Ao final de dois anos, no grupo da predniso-

lona, 25,9\% dos pacientes apresentaram progressão das lesões radiográficas; no grupo sem prednisolona essa taxa foi de $39,3 \%(p=0,033)$. Aos dois anos, $55 \%$ dos pacientes do primeiro grupo alcançaram remissão (D AS28<2,6) em comparação com $32,8 \%$ do outro grupo $(p=0,0005)$. A perda óssea durante os dois anos foi similar entre os grupos. O s autores concluem que a adição de baixa dose de prednisolona ao tratamento inicial com DMARD em pacientes com AR inicial reduz a progressão de dano articular, controla a inflamação, melhora a capacidade física e é bem tolerada.

Van der H eijde D, Klareskog L, Rodríguez-Valverde V et al: Comparison of etanercept and methotrexate, alone and combined, in treatment of rheumatoid arthritis (C omparação entre etanercepte e M TX, sozinhos e combinados, no tratamento da AR). Arthritis Rheum 54:1063-74, 2006. Department of Rheumatology, U niversity $\mathrm{H}$ ospital, $\mathrm{M}$ aastricht, $\mathrm{H}$ olanda.

$\mathrm{O}$ artigo revela os resultados do segundo ano do estudo TEM PO, desenhado como um ensaio clínico, duplo-cego e randomizado para avaliar a eficácia e segurança do uso de etanercepte e M TX em monoterapia e em terapia combinada. O s 503 pacientes que completaram o primeiro ano seguiram divididos recebendo etanercepte na dose de 25 mg, subcutâneo, duas vezes por semana, M TX até $20 \mathrm{mg}$, via oral, por semana (dose média de $16,5 \mathrm{mg}$ ) ou a combinação das duas drogas. As respostas ACR 50 e 70 foram alcançadas por $71 \%$ 49\% no grupo etanercepte + M TX, $42 \% 21 \%$ no grupo M TX e $54 \%$ d $27 \%$ no grupo etanercepte, apresentando diferença estatisticamente significativa em favor da terapia combinada em relação às monoterapias $(p<0,01)$. A terapia combinada também foi superior, levando a melhores resultados de D AS e D AS28. 0 Escore de Sharp Total ( Total Sharp ScoreTSS) e o número de erosões foram menores no grupo etanercepte + M TX quando comparado aos grupos de monoterapia $(p<0,05)$. 0 grupo combinado obteve uma diferença média de TSS entre o valor basal e o valor final do primeiro e segundo anos menor que zero (regressão radiológica), demonstrando que a terapia combinada foi capaz de reparar dano estrutural neste grupo de pacientes. Em relação à toxicidade eao número de efeitos colaterais, não houve diferença entre os três grupos. 
M oreland L, Weinblatt M E, Keystone EC et al: Etanercept treatment in adults with established rheumatoid arthritis: 7 years of clinical experience (Tratamento com etanercepte em adultos com artrite reumatóide estabelecida: 7 anos de experiência clínica). J R heumato/ 33: 5-12, 2006. U niversity of Alabama at Birmingham, Birmingham, Alabama, EU A.

O objetivo deste estudo foi avaliar segurança, melhora de função e de qualidade de vida com o uso de etanercepte a longo prazo. Foram selecionados 714 pacientes com AR refratária ao tratamento com DMARDs, que receberam etanercepte em algum dos sete estudos iniciais ou em uma extensão. 0 tratamento individual mais longo foi de 8,2 anos. Ao final do primeiro ano, 560 pacientes foram analisados, obtendo-se as seguintes respostas: ACR20 70\%, ACR50 - 44\%, ACR 70 - 19\%; DAS28<3,2 - 37\%e DAS28 $<2,6-23 \%$ Ao final de seis anos, 356 pacientes foram analisados, observando-se as seguintes taxas: ACR 20
- 73\%; ACR50 - 52\% ACR 70 - 27\%; D AS28<3,2- 47\% e DAS28 <2,6- 36\% Ao final de sete anos, 167 pacientes foram analisados e as respostas foram semelhantes. As freqüências de eventos adversos sérios (14,8/ 100 pacientes-ano), infecções graves (4,2/ 100 pacientes-ano), câncer ( $1,0 / 100$ pacientes-ano) e mortes (0,7/ 100 pacientes-ano) foram estáveis a cada ano. Os autores acreditam que os efeitos adversos encontrados foram compatíveis com os esperados e que o tratamento com etanercepte em pacientes adultos com AR mostra resposta clínica rápida que se sustenta por sete anos ou mais.

$\mathrm{H}$ araoui B, Cameron L, O uellet M, White B: Anti-infliximab antibodies in patients with rheumatoid arthritis who require higher doses of infliximab to achieve or maintain a clinical response (Anticorpos anti-infliximabe em pacientes com AR que necessitam doses maiores de infliximabe para alcançar ou manter resposta clínica). J Rheumato/ 33: 31- 6, 2006. Department of R heumotology, Centre H ospitalier de L 'U niversité de M ontréal, and Groupe de reserche de maladies rhumatismales du Q uébec, M ontreal, Q uebec, C anadá.

Os autores tiveram como objetivo determinar se a necessidade do uso de doses de infliximabe maiores que $3 \mathrm{mg} / \mathrm{kg}$ a cada oito semanas para atingir e manter resposta clínica em pacientes com AR está associada a diferenças em características clínicas de base ou a anticorpos antiinfliximabe. Foram avaliados, retrospectivamente 51 , pacientes, divididos em dois grupos: grupo 1 ( $n=19 ; 37 \%)$ - pacientes que atingiram e mantiveram resposta clínica com doses usuais; grupo 2 ( $n=32 ; 63 \%)$ - pacientes que necessitaram de doses maiores. Anticorpos antiinfliximabe ocorreram em $47 \%$ dos pacientes do grupo 2 e em $27 \%$ do grupo 1, porém essa diferença não foi estatisticamente significativa. Concentrações significativamente mais altas foram observadas no grupo $2(p=0,02)$. O s pacientes que desenvolveram anticorpos antiinfliximabe eram, em média, 10 anos mais jovens e estavam usando doses menores de prednisona no período de infusão do infliximabe. $\mathrm{N}$ ão foram identificadas outras diferenças clínicas entre os grupos. O s autores acreditam que possa haver uma relação entre maiores concentrações de anticorpos antiinfliximabe e a necessidade de doses maiores de infliximabe em pacientes com AR .

Breedveld FC, Weisman $\mathrm{MH}$, Kavanaugh AF et al: The PREMIER study: a multicenter, randomized, double-blind clinical trial of combination therapy with adalimumab plus methotrexate versus methotrexate alone or adalimumab alone in patients with early, aggressive rheumatoid arthritis who had not had previous methotrexate treatment (Estudo PREM IER: um ensaio clínico, multicêntrico, randomizado, duplo-cego de terapia combinada com adalimumabe mais metotrexato versus metotrexato isolado ou adalimumabe isolado em pacientes com AR inicial e agressiva, sem tratamento prévio com metotrexato). Arthritis Rheum 54: 26-37, 2006. Leiden U niversity M edical Centre, Leiden, $\mathrm{H}$ olanda.

O s autores compararam a eficácia e segurança da terapia combinando adalimumabe e M TX oral versus monoterapia com MTX oral ou monoterapia com adalimumabe, em pacientes com AR precoce ( $<3$ anos de evolução). Foram selecionados 799 pacientes com critérios de gravidade e que nunca haviam recebido, previamente, drogas modifi- cadoras da doença. A dose média de M TX empregada foi de aproximadamente $16 \mathrm{mg} /$ semana. 0 adalimumabe foi usado na dose de $40 \mathrm{mg}$, subcutâneo, a cada duas semanas. O s objetivos primários avaliados após um ano foram ACR 50 ealteração média da radiografia de base. A resposta ACR 50 foi alcançada após um ano em $62 \%$ dos pacientes em 
terapia combinada e em $41 \%$ e $46 \%$ dos grupos que receberam, respectivamente, adalimumabe e M TX em monoterapia. Tendência semelhante foi observada para ACR 50 em 2 anos e para ACR 20/ 70/ 90 em um e dois anos. Em relação à progressão radiológica em 6, 12 e 24 meses, constatou-se significativa redução com a terapia dupla comparada às monoterapias e diferença estatística favorecen do adalimumabe versus M TX, quando administrados sozinhos $(p<0,001)$. A remissão clínica (definida como D AS28 menor que 2,6 ) foi duas vezes mais freqüente com a terapia dupla $(p<0,001)$. Os efeitos adversos foram similares nos três grupos. 0 estudo demonstrou superioridade da terapia combinada sobre as monoterapias para a resposta clínica e radiológica e superioridade do adalimumabe sobre o M TX, ambos em monoterapia, para redução da progressão radiológica em AR inicial e agressiva.

E dwards JCW, Szczepanski L, Szechinski J et al: Efficacy of B-cell-targeted therapy with rituximab in patients with rheumatoid arthritis (Eficácia da terapia anticélulas-B com rituximabe em pacientes com AR.) $N$ Engl J M ed 350: 2572-81, 2004. U niversity College London, Londres, Reino U nido.

Este estudo duplo-cego analisou os resultados do tratamento da AR soro-positiva em pacientes com aproximadamente dez anos de doença, uso prévio de uma a quatro drogas modificadoras da doença mantendo atividade inflamatória moderada a grave. U m total de 161 pacientes foi randomisado para receber M TX oral (grupo controle, $n=40)$, rituximabe e $M T X(n=40)$, rituximabe e ciclofosfamida ( $n=41$ ) ou rituximabe em monoterapia ( $n=40$ ). As doses médias de MTX foram de 12,5 a 15 $\mathrm{mg} /$ semana, a ciclofosfamida foi dada em duas infusões (dias 3 e 17) de $750 \mathrm{mg}$ e o rituximabe em duas infusões de $1000 \mathrm{mg}$ (dias 1 e 15). Todos os pacientes receberam corticosteróides inicialmente. A resposta ACR 50 após 24 semanas (desfecho primário) foi mais freqüente nos grupos de rituximabe combinado à M TX (41\%) e com ciclofosfamida (43\%), respectivamente, versus M TX isolado
( $13 \%, p=0,005)$. Rituximabe em monoterapia não produziu resposta ACR50 diferente do grupo controle (33\% $p=0,059)$. Após 48 semanas, $35 \%$ do grupo rituximab associado à MTX e $27 \%$ do grupo rituximab e ciclofosfamida ainda mantinham os critérios de melhora ACR 50 $(p=0,002$ e $p=0,01$, respectivamente). A penas a combinação de rituximabe com MTX produziu respostas ACR 70 estatisticamente significativas após 24 e 48 semanas (23\%, $p=0,048$ e $15 \%, p=0,03$, respectivamente). O bservou-se também diminuição prolongada na população delinfócitos $B$, sem queda significativa dos níveis de imunoglobulinas ou de anticorpos antitétano. O s autores mostraram que a terapia de curto prazo com rituximabe pode produzir respostas duradouras especialmente quando combinada ao MTX, confirmando então um papel importante das células $B$ na fisiopatologia da AR.

Genovese M C, Becker JC, Schiff M, et al: Abatacept for rheumatoid arthritis refractory to tumor necrosis factor inhibition (A batacepte para AR refratária à inibição do fator de necrose tumoral). N EnglJ M ed 353:11, 2005. Stanford U niversity M edical Center, Stanford, Califórnia, EU A.

O s autores conduziram este estudo duplo-cego com o objetivo de avaliar a eficácia e a segurança do abatacepte em pacientes com AR ativa e resposta inadequada à terapia com inibidores de TN F, após pelo menos três meses de tratamento. Os pacientes foram randomizados em dois grupos: o primeiro recebeu abatacepte combinado com pelo menos uma DMARD $(n=258)$ e outro grupo recebeu D M ARD e placebo como grupo-controle $(n=133)$. D uzentos e vinte e três pacientes completaram seis meses de acompanhamento no grupo em uso de abatacepte e 99, no grupo controle. Após esse período de seguimento, os autores encontraram taxas de resposta ACR 20 de $50,4 \%$ e de 19,5\% ( $p<0,001$ ) nos grupos abatacepte e controle, respectivamente. As taxas de resposta ao ACR50 e ACR70 também foram maiores no grupo em uso de abatacepte. Houve melhor resposta funcional, avaliada pelo HAQ no grupo em uso de abatacepte $(p<0,001)$. A incidência de efeitos adversos foi semelhante nos dois grupos. Os autores concluem que, no período estudado, o abatacepte mostrou-se clinicamente eficaz e seguro em pacientes com AR e resposta inadequada à terapia com anti-TN F. 
O arquivo disponível sofreu correções conforme ERRATA publicada no Volume 46 Número 4 da revista. 